\title{
IGEMS: The Consortium on Interplay of Genes and Environment across Multiple Studies
}

\author{
Nancy L. Pedersen ${ }^{1,2}$, Kaare Christensen ${ }^{3}$, Anna K. Dahl ${ }^{1,10}{ }^{\text {, Deborah Finkel }}{ }^{4}$, Carol E. \\ Franz $^{5}$, Margaret Gatz ${ }^{1,2}$, Briana N. Horwitz ${ }^{12}$, Boo Johansson ${ }^{6}$, Wendy Johnson ${ }^{7}$, William \\ S. Kremen ${ }^{5,8}$, Michael J. Lyons ${ }^{9}$, Bo Malmberg ${ }^{10}$, Matt McGue ${ }^{11,3}$, Jenae M. Neiderhiser ${ }^{12}$, \\ Inge Petersen ${ }^{3}$, and Chandra A. Reynolds ${ }^{13}$ \\ ${ }^{1}$ Department of Medical Epidemiology and Biostatistics, Karolinska Institutet, Stockholm, Sweden \\ ${ }^{2}$ Department of Psychology, University of Southern California, Los Angeles, CA, USA \\ ${ }^{3}$ The Danish Twin Registry, University of Southern Denmark, Institute of Public Health, \\ Epidemiology, Odense C, Denmark \\ ${ }^{4}$ Department of Psychology, Indiana University Southeast, New Albany, IN, USA \\ ${ }^{5}$ Department of Psychiatry, University of California - San Diego, La Jolla, CA, USA \\ ${ }^{6}$ Department of Psychology, University of Gothenburg, Gothenburg, Sweden \\ ${ }^{7}$ Department of Psychology and Centre for Cognitive Ageing and Cognitive Epidemiology, \\ University of Edinburgh, Edinburgh, UK \\ ${ }^{8}$ Center of Excellence for Stress and Mental Health, VA San Diego Healthcare Center, La Jolla, \\ CA, USA \\ ${ }^{9}$ Department of Psychology, Boston University, Boston, MA, USA \\ ${ }^{10}$ Institute of Gerontology, School of Health Sciences, Jönköping University, Jönköping, Sweden \\ ${ }^{11}$ Department of Psychology, University of Minnesota, Minneapolis, MN, USA \\ ${ }^{12}$ Department of Psychology, Penn State University, University Park, PA, USA \\ ${ }^{13}$ Department of Psychology, University of California - Riverside, Riverside, CA, USA
}

\begin{abstract}
The Interplay of Genes and Environment across Multiple Studies (IGEMS) group is a consortium of eight longitudinal twin studies established to explore the nature of social context effects and gene-environment interplay in late-life functioning. The resulting analysis of the combined data from over 17,500 participants aged 25-102 at baseline (including nearly 2,600 monogygotic and 4,300 dizygotic twin pairs and over 1,700 family members) aims to understand why early life adversity, and social factors such as isolation and loneliness, are associated with diverse outcomes including mortality, physical functioning (health, functional ability), and psychological functioning (well-being, cognition), particularly in later life.
\end{abstract}

(C) The Authors 2012

Address For Correspondence: Nancy L. Pedersen, Department of Medical Epidemiology and Biostatistics, Karolinska Institutet, Box 281, SE-171 77 Stockholm, Sweden. Nancy.Pedersen@ki.se. 


\section{Keywords}

twins; gene-environment interaction; aging; longitudinal

Epidemiological research has firmly established the association of social context with latelife health and functioning (Cacioppo et al., 2006; Cohen, 2004). Yet this research unambiguously explicates neither the basis for these associations nor how social context relates to the biological and genetic factors known to contribute to later life functioning (Cohen \& Janicki-Deverts, 2009; Hawkley \& Cacioppo, 2003). Several longitudinal twin samples, located in different countries and employing somewhat different measures, have individually accumulated substantial data. Because recruiting and maintaining long-running studies of older adults is made especially difficult by participants' relative frailty, these samples have generally been too small to reliably detect many of the kinds of effects likely to be of interest. Consequently, a collaboration among eight existing longitudinal twin and family studies was initiated, with a central focus on determining how social context is related to physical functioning (health, functional ability), and psychological functioning (well-being, cognition) in mid-life and older ages. Through this, we hope to lay the foundation for future studies of gene-environment interplay in late-life functioning. In the following we describe the rationale and design of the Interplay of Genes and Environment across Multiple Studies (IGEMS) consortium.

With their rich registry systems, the Nordic countries have pioneered many of the major longitudinal twin studies of aging, including the Longitudinal Study of Aging Danish Twins (LSADT) in Denmark (Christensen et al., 1999) the Swedish Adoption Twin Study of Aging (SATSA; Finkel \& Pedersen, 2004), Origins of Variance in the Old-Old (OCTO-Twin; McClearn et al., 1997), and Ageing in Women and Men: A Longitudinal Study of Gender Differences in Health Behaviour and Health among Elderly (Gender; Gold et al., 2002) projects in Sweden. Moreover, investigators in both countries have extended their investigations to younger adult twin samples (e.g., the Middle-Age Danish Twin [MADT] study; Osler et al., 2008) and Twin and Offspring Study in Sweden (TOSS; Neiderhiser \& Lichtenstein, 2008), to provide foundations for longitudinal investigations of the influence of earlier developmental periods on aging outcomes. While undertaking similar longitudinal twin research in the United States has been more logistically challenging, members of IGEMS have established analogous studies in the United States, most notably the Minnesota Twin Study of Adult Development and Aging (MTSADA; Finkel \& McGue, 1993) and the Vietnam Era Twin Study of Aging (VETSA; Kremen et al., 2006). Collectively, these studies include over 17,500 participants (including nearly 2,600 monogygotic [MZ] and 4,300 dizygotic [DZ] twin pairs and over 1,700 family members) whose ages ranged from 25 years to over 100 years at their intake assessments. Participants in these studies have undergone multiple longitudinal assessments (median follow-up period $=9.5$ years) that include a rich variety of measures of aging-relevant outcomes in three broad domains: physical health and functional ability, psychological well-being (emotional stability/ depression), and cognitive health. These studies also include multiple indicators of the social environment, spanning early childhood through late adulthood, and encompass participants with several different environmental contexts, such as differences in healthcare and retirement systems. The combined result is a set of longitudinal studies with assessments of outcomes and social contexts spanning mid- to late-adulthood.

The heuristic model that guides our investigation of gene-environment interplay in late-life functioning is presented in Figure 1. The model emphasizes the importance of two geneenvironment processes. First, we hypothesize that environmental exposures and social contexts do not occur at random, but rather reflect an individual's genetically influenced 
behavior and choices (i.e., gene-environment correlation). We know, for example, that there are heritable influences on social factors including social support (Bergeman et al., 2001), social engagement (McGue \& Christensen, 2007), and social isolation (Boomsma et al., 2007). We further hypothesize that gene-environment correlations are a major contributor to late-life phenotypic stability. For example, based on Schmalhausen (1946), we hypothesize that a shift from being socially engaged and physically active in middle age to being socially isolated in late-life will be accompanied by marked discontinuities in behavioral stability. Data in the IGEMS consortium allow us to chart the contributions of changes in social context to behavioral discontinuities; the twin-study design allows us to further explore the nature of gene-environment interplay in these transitions.

Second, we hypothesize that experiential factors can either diminish or amplify the influences of genetic effects on late-life outcomes (genotype-environment interaction, $\mathrm{G} \times$ E). Two of Shanahan and Hofer's (2005) four alternative models of $\mathrm{G} \times \mathrm{E}$ are directly relevant. Evolutionary models (Schmalhausen, 1946) predict that the $\mathrm{G} \times \mathrm{E}$ form termed Contextual Triggering by Shanahan and Hofer, but recognized more broadly as the diathesis-stress model, will be more pronounced in late life. Specifically, during most of life, the typical human lives in an environment that is low in stress relative to the environments in which their species evolved (Owens, 2002). In old age, biological, psychological, and social stresses are considered to be higher due to more negative change or declines. Thus, at times of relatively increased stress—-such as old age—an organism's response to an unfavorable environment will depend on a unique combination of genes, so that genetic effects on physical manifestations of health will be maximized (Schmalhausen, 1946). This pattern of findings is supported by earlier work by IGEMS investigators, showing that the heritability of physical health in mid-life is maximized when current income (Johnson \& Krueger, 2005a) and perceived life control (Johnson \& Krueger, 2005b) are low.

Alternatively, environmental factors can suppress genetic effects through a form of $\mathrm{G} \times \mathrm{E}$ Shanahan and Hofer call Social Context as Social Control. We predict that the Social Context as Social Control form of $\mathrm{G} \times \mathrm{E}$ is particularly relevant to extreme forms of physical disability or disease, which diminish the impact of genetic factors by reducing the opportunity for self-selection of activities that maintain stable functioning.

\section{IGEMS Sample}

Eight studies are involved in the IGEMS consortium. Brief descriptions of the studies are provided here and sample sizes are summarized in Table 1.

\section{Swedish Studies}

Four twin studies based in Sweden are included in IGEMS. The SATSA (Finkel \& Pedersen, 2004) is a longitudinal twin study that began in 1984. Multiple measures of physical and psychological functioning and environmental/social context variables were assessed via interviews and questionnaires. Eight questionnaires have been sent to all study participants. Mean age at the first questionnaire assessment was $60.14(S D=14.02), 42.1 \%$ male, and all are Caucasians. Mean level of attained education was 8 years $(S D=2.38$; range 6-16). Nine waves of in-person testing of twins over the age of 50 have been completed; the tenth wave is currently on going. The base population comprises all pairs of twins from the population-based Swedish Twin Registry, who indicated that they had been separated before the age of 10 and reared apart; and a sample of twins reared together, matched on the basis of gender, and date and county of birth. Individuals were included in the longitudinal study whether or not both members of the pair responded, and those who did not respond at the first wave were invited at subsequent waves. Zygosity was initially based on questions concerning similarity during childhood, administered when the Swedish 
Twin Registry was first compiled. These assignments were refined for participants in the first in-person testing-based serological markers, and further refined after genotyping (most recently with the cardio-metabochip).

The OCTO-Twin Study was the first major population-based longitudinal twin study directed to the oldest old (McClearn et al., 1997). The OCTO-Twin Study included five measurement occasions at 2-year intervals between 1991 and 2002 of 351 like-sexed twin pairs drawn from the Swedish Twin Registry who were over the age of 80 at baseline. Mean age at baseline assessment was $83.58(S D=3.17), 33.3 \%$ male, and all are Caucasians. Inperson testing included assessment of physical and psychological functioning, and environmental/social context variables. The assessments were built on those from SATSA. Only pairs where both responded at baseline are included. Mean level of attained education was 7.09 years $(S D=2.24$; range $0-25)$. Like other Swedish twin studies, zygosity was initially based on registry-based responses to questions regarding similarity. For those pairs with undetermined zygosity at the start of OCTO-Twin, zygosity was evaluated through Restriction Fragment Length Polymorphism (RFLP) markers. Like SATSA, some correction in zygosity has been made as further genotyping with the cardio-metabochip has become available.

Gender is a study of unlike-sex twin pairs born between 1906 and 1925 (Gold et al., 2002). A survey concerning health and health behavior was mailed in 1994 with responses from 1,210 twins from 605 pairs where both responded. Mean age at baseline questionnaire assessment was $74.43(S D=4.28)$ and all are Caucasians. Mean level of attained education was 7.28 years $(S D=2.4$; range 5-25). A baseline in-person evaluation of 498 twins from 249 pairs between 70 and 80 years of age was undertaken between 1995 and 1997, and included an interview and tests of cognitive and physical functioning, similar to SATSA and OCTO-Twin. Two additional in-person waves followed at 4-year intervals. A baseline inperson evaluation of pairs 80 years and older took place between 1998 and 2002 with an interview and tests of cognitive and physical functioning similar to OCTO-Twin. Finally, a second survey was mailed in 2007 to all living twins who participated in the first mailed survey.

The TOSS was designed to examine how family relationships influence the adjustment of adult twins and their family members, how genetic and environmental factors may explain these associations, and to better specify genotype-environment correlation (Neiderhiser \& Lichtenstein, 2008). TOSS includes 909 pairs of same-sex twins, one adolescent child of each twin, and the spouse/partner of each twin. The average age is 47 ( $S D=4.7$ years, range $35-59)$ for the 350 male twin pairs and ( $S D=4.6$ years, range 32-60) for the 559 female twin pairs. Mean level of attained education was 13.28 years $(S D=2.74$; range 9-16). Zygosity was determined by analyses of markers from DNA extracted from mouthwash or saliva samples. For the 54 twins who did not provide DNA, zygosity was determined by a questionnaire assessing twin similarity and by applying an algorithm base on a discriminant analysis of twins with DNA-confirmed zygosity. There were $254 \mathrm{MZ}$ and $285 \mathrm{DZ}$ twin mother pairs and $128 \mathrm{MZ}$ and $183 \mathrm{DZ}$ twin father pairs. The TOSS included a single assessment that indexed psychological functioning and extensive environmental/social context variables; however, longitudinal information is available from STR assessments. Only the twins and their spouses are included in the IGEMS.

\section{Danish Studies}

Two Danish studies are included in IGEMS. The LSADT (Christensen et al, 1999) began in 1995 with the assessment of members of like-sex twin pairs born in Denmark prior to 1920 (i.e., at least 75 years old). The surviving members of the initial cohort were followed up every 2 years in 1997, 1999, 2001, 2003, and 2005. Additional cohorts were added in 1997, 
1999, and 2001 and the minimum age requirement was progressively reduced to age 70. Mean level of attained education was 8.65 years $(S D=2.87$; range $5-18)$. Mean age at intake was 77.26 years $(S D=5.65$; range $70-102)$ and $41 \%$ were males. Multiple measures of physical and psychological functioning and environmental/social context variables were assessed.

The study of MADT includes twins ranging in age from 46 to 68 years at the original assessment from 1998 to 1999 (Osler et al., 2008). Mean level of attained education was 11.10 years $(S D=2.96$; range $7-18)$. Mean age at intake was 56.43 years $(S D=6.33)$ and $51 \%$ were males. A second assessment of MADT participants began in 2009. The MADT assessment closely parallels that used in LSADT.

The participants of LSADT and MDAT surveys are all Caucasians. Determination of zygosity in LSADT as well as MADT is based on the twins' responses to four questions on physical similarity, a method that has previously been demonstrated to assign the true zygosity in more than 95\% of the twin pairs (Christiansen et al., 2003).

\section{US Studies}

The MTSADA (Finkel \& McGue, 1993; McGue et al., 1993) is a population-based sample drawn from state birth records. Although MTSADA has a broad age range, two-thirds of the sample was age 60 years or older at intake (mean 58.6 years, $S D=10.8$ ). Mean level of attained education was 13.31 years $(S D=2.61$; range $0-25), 42 \%$ were males, and $98.5 \%$ were Caucasians, consistent with the demographics of Minnesota for the birth cohorts sampled. Participation involved two interview sessions separated by 1-3 weeks at which multiple physical functioning, psychological functioning, and environmental/social context variables were assessed. Interviews took place between 1984 and 1994. The MTSADA sample completed a follow-up mail survey approximately 6 years after initial assessment. Zygosity was assessed using five questions dealing with physical similarity, which previous research has shown yields approximately $95 \%$ accuracy when compared to genetic-based methods (Lykken et al., 1990).

The VETSA (Kremen et al., 2006) is a community-dwelling sample of male-male twin pairs, all of whom served in some branch of US military service at sometime between 1965 and 1975. The majority were not in combat. They now live throughout the United States and are generally comparable in terms of demographic and health characteristics to American men in their age range (Schoeneborn \& Heyman, 2009). Wave 1 interviewing took place between 2003 and 2007. The design is somewhat unusual in that the VETSA has a rather narrow age range. At the time of recruitment for the Wave 1 assessment, all participants were in their 50 s (although two pairs turned 60 by the time they came to the laboratory). The mean level of formal education completed was 13.84 years $(S D=2.11$; range: $8-20)$. Eighty-six percent were Caucasian. Zygosity was determined on the basis of 25 microsatellite markers for $92 \%$ of the sample, and by questionnaire and blood group information for the remaining $8 \%$. The latter method agreed with the DNA-based results in $95 \%$ of cases. Both twins in a pair had to agree to participate in order to be enrolled in Wave 1 , but there is no such requirement for follow-up assessments. Participants filled out multiple questionnaires at home about physical and psychological functioning, lifestyle, and environmental/social context. They also underwent a full day of in-person assessments (in San Diego or Boston according to participant preference) focused on cognitive and physical function. Participants who could or would not come to one of the laboratories were tested in their hometowns. Assessments are currently planned for every 5 years, and the Wave 2 assessment is nearly complete (as of September 2012) with a retention rate of approximately $85 \%$. A key goal of the VETSA is to make use of its age-homogenous cohort to understand individual differences in aging trajectories in the transition from mid- to later life. 


\section{IGEMS Measures}

There is a wealth of data available from the eight studies, predominantly from the series of measurement occasions within each longitudinal study. Table 2 shows the key domains from the heuristic model and the measures available across the eight studies to assess those domains. As indicated in the table, these measures were largely obtained by mailed questionnaire or interview, with some physical or functional variables measured during inperson visits. In addition to these data, national registry data available in Sweden and Denmark allow us to obtain supplemental objective measures of health and early environment (such as birth weight). Furthermore, the twin registries also have information provided by the twins when the registries were compiled (in the 1960s and 1970s).

\section{Data Harmonization}

Given the differences in study focus, design, and specific measures assessed, we have placed strong emphasis on data harmonization, to ensure the greatest possible consistency of measurement across studies. We developed a common administrative file structure that includes demographic variables, last known vital status, age at each time of assessment, and reasons for non-participation if the twin did not participate at that wave. Because of interstudy differences in how cohorts were defined and schedule of data collection, it was crucial to come to common definitions that would allow combining studies longitudinally.

At the same time, we undertook harmonization of relevant phenotypes and outcomes to enable the combined analysis of the multiple sets of data. We are not simply recoding to a reduced set of common categories, but rather are identifying overlapping item content and response formats, applying psychometric analysis to establish measurement invariance via item response theory (IRT)-factor analytic approaches, and conducting meta-analyses and integrated data analysis of pooled data where warranted (Bauer \& Hussong, 2009; Curran \& Hussong, 2009; Rosenthal, 1991; Rosenthal \& Di Matteo, 2001).

As a first step, we created spreadsheets for measures that correspond to the constructs in the model, including the specific questions and response options. Table 2 indicates which variables were available; detailed spreadsheets show how each variable was assessed (see example in Figure 2).

Because integrative data analysis (IDA), where data are pooled across samples to increase power and finely consider between-study heterogeneity, is the preferred method (Curran \& Hussong, 2009), we are creating scale scores that are common across studies. This requires overlapping item content across the studies as well as across time for longitudinal hypotheses. Thus, a second step entailed identifying those measures for which it was possible to identify a common metric; for example, Body Mass Index (BMI), lung function, and blood pressure. For harmonizing education, we have created year equivalents for educational levels among Danish and Swedish studies that did not collect the data in years.

Where a common metric is not already available, IRT techniques are implemented to create harmonized scores across studies (cf. Bauer \& Hussong, 2009). The premise is that trait scores are estimable based on individual responses to and features of items (e.g., item 'difficulty', discrimination and reliability; Embretson \& Reise, 2000). Moreover, in the case of IDA, corresponding indices are available across samples with items that invariantly tap a particular construct(s) (Bauer \& Hussong, 2009). Multiple common items should be available to test that item functioning is similar across samples (Bauer \& Hussong, 2009), and to establish 'crosswalks' between the different measurement indices (e.g., Velozo et al., 2007). 
For example, all Swedish studies (SATSA, OCTO-Twin, Gender, TOSS) and the US studies (MTSADA and VETSA) measured depressive symptomatology using the Center for Epidemiologic Studies - Depression scale (CES-D) (Radloff, 1977), while the Danish studies used an adapted version of the Cambridge Mental Disorders of the Elderly Examination (CAMDEX; see McGue \& Christensen, 1997). There was no item with the same wording and the same response format on the two scales. However, both scales appeared to encompass the same conceptualization of depression. The CES-D can be analyzed as a single score or factored into four sub-scales (Gatz et al., 1993): depressed mood, somatic complaints, interpersonal difficulties, and lack of well-being. The 21

CAMDEX items can be analyzed as a single score or as two-factor analytically derived subscales: an affect sub-scale and a somatic sub-scale. Substantially similar results have been reported across studies with respect to the relative roles of genetic and environmental influences on depressive symptoms (Gatz et al., 1992; McGue \& Christensen, 1997) and the possibility that heritability may be greater in women than men (Jansson et al., 2004; McGue \& Christensen, 1997). Since we could find no published article where the same sample had taken both measures, as a third step in data harmonization, we recruited a separate new sample and administered both scales to that sample, after which it was possible to use IRT methods to establish a 'crosswalk' to apply to the scores to create a common scale.

We also used this approach with perceived socioeconomic status (SES) and self-rated health items, where the variations on how each was assessed were extensive across studies. Similar to the depression example, we have used IRT modeling to calibrate a common metric for a subset of perceived SES items measured in three Swedish studies (SATSA, OCTO-Twin, and Gender) and the Minnesota study (MTSADA) by using recruitment samples that were administered a subset of items in common among and unique to the Swedish and Minnesotan samples. Specifically, we evaluated a total of six items from the Swedish and Minnesota studies. Examples of perceived SES items included: (1) 'Do you have difficulty in meeting general expenses like food, rent, bills etc?' (No/Yes); (SATSA, Gender); (2) 'I have enough finances to pay monthly bills' ( Yes/No); (MTSADA). Additional recruitment samples will be given full sets of perceived SES items across all available Swedish and US samples, adding items from TOSS and VETSA.

In a third example, we have examined similar self-rated health items included in all eight studies. While item prompts are similar, response metrics varied significantly, ranging from two to as many as seven response options. All combinations of items and response options were collected from an independent sample, and these data were used to develop latent selfrated health factors that could be combined across studies.

At the end of this process, we will have a common set of measures, each from as many studies as possible, to pursue testing the heuristic model presented above. Where integrative analyses cannot be used, we are relying on meta-analytic methods (Rosenthal, 1991; Rosenthal \& Di Matteo, 2001), using fixed- and random-effects approaches where appropriate.

\section{Conclusion}

The expansion of the elderly population has brought with it challenges for healthcare and social delivery systems, which may become overburdened with large numbers of disabled and cognitively impaired individuals (Christensen et al., 2009). There is a clear need to understand the basis for healthy aging and especially to identify and characterize the nature of the modifiable risk and protective factors associated with later-life functioning (Christensen et al., 2008). IGEMS will support a series of epidemiological, genetic, and 
biological investigations of one class of potentially modifiable factors, those related to social context.

\section{Acknowledgments}

The IGEMS consortium acknowledges the contributions of Chris J. Hahn, Marielena De Araujo-Held, and Catalina Zavala. Most importantly, the authors gratefully acknowledge the continued cooperation and participation of the twins and their families.

IGEMS is supported by the National Institutes of Health grant no. R01 AG037985. SATSA was supported by grants R01 AG04563, R01 AG10175, the MacArthur Foundation Research Network on Successful Aging, the Swedish Council for Working Life and Social Research (FAS) (97:0147:1B, 2009-0795) and Swedish Research Council (825-2007-7460, 825-2009-6141). OCTO-Twin was supported by grant R01 AG08861. Gender was supported by the MacArthur Foundation Research Network on Successful Aging, The Axel and Margaret Ax:son Johnson's Foundation, The Swedish Council for Social Research, and the Swedish Foundation for Health Care Sciences and Allergy Research. TOSS was supported by grant R01 MH54610 from the National Institute of Health. The Danish Twin Registry is supported by grants from The National Program for Research Infrastructure 2007 from the Danish Agency for Science and Innovation, the Velux Foundation and the US National Institute of Health (P01 AG08761). The Minnesota Twin Study of Adult Development and Aging was supported by NIA grant R01 AG 06886. VETSA was supported by National Institute of Health grants R01 AG018384, R01 AG018386, R01 AG022381, and R01 AG022982, and, in part, with resources of the VA San Diego Center of Excellence for Stress and Mental Health. The Cooperative Studies Program of the Office of Research \& Development of the United States Department of Veterans Affairs has provided financial support for the development and maintenance of the Vietnam Era Twin (VET) Registry.

\section{References}

Bauer DJ, Hussong AM. Psychometric approaches for developing commensurate measures across independent studies: Traditional and new models. Psychological Methods. 2009; 14:101-125. [PubMed: 19485624]

Bergeman CS, Neiderhiser JM, Pedersen NL, Plomin R. Genetic and environmental influences on social support in later life: A longitudinal analysis. International Journal of Aging \& Human Development. 2001; 53:107-135. [PubMed: 11758722]

Boomsma DI, Cacioppo JT, Muthen B, Asparouhov T, Clark S. Longitudinal genetic analysis for loneliness in Dutch twins. Twin Research and Human Genetics. 2007; 10:267-273. [PubMed: 17564516]

Cacioppo JT, Hughes ME, Waite LJ, Hawkley LC, Thisted RA. Loneliness as a specific risk factor for depressive symptoms: Cross-sectional and longitudinal analyses. Psychology and Aging. 2006; 21:140-151. [PubMed: 16594799]

Christensen K, Doblhammer G, Rau R, Vaupel JW. Ageing populations: The challenges ahead. Lancet. 2009; 374:1196-1208. [PubMed: 19801098]

Christensen K, Holm NV, McGue M, Corder L, Vaupel JW. A Danish population-based twin study on general health in the elderly. Journal of Aging and Health. 1999; 11:49-64. [PubMed: 10848141]

Christensen K, McGue M, Petersen I, Jeune B, Vaupel JW. Exceptional longevity does not result in excessive levels of disability. Proceedings of the National Academy of Sciences of the United States of America. 2008; 105:13274-13279. [PubMed: 18711139]

Christiansen L, Frederiksen H, Schousboe K, Skytthe A, von Wurmb-Schwark N, Christensen K, Kyvik K. Age- and sex-differences in the validity of questionnaire-based zygosity in twins. Twin Research. 2003; 6:275-278. [PubMed: 14511432]

Cohen S. Social relationships and health. American Psychologist. 2004; 59:676-684. [PubMed: 15554821]

Cohen S, Janicki-Deverts D. Can we improve our physical health by altering our social networks? Perspectives on Psychological Science. 2009; 4:375-378. [PubMed: 20161087]

Curran PJ, Hussong AM. Integrative data analysis: The simultaneous analysis of multiple data sets. Psychological Methods. 2009; 14:81-100. [PubMed: 19485623]

Embretson, SE.; Reise, SP. Item response theory for psychologists. Mahwah, NJ: Lawrence Erlbaum Associates; 2000. 
Finkel D, McGue M. The origins of individual differences in memory among the elderly: A behavior genetic analysis. Psychology and Aging. 1993; 8:527-537. [PubMed: 8292281]

Finkel D, Pedersen NL. Processing speed and longitudinal trajectories of change for cognitive abilities: The Swedish Adoption/Twin Study of Aging. Aging, Neuropsychology, and Cognition Special Issue: Longitudinal Studies Of Cognitive Aging. 2004; 11:325-345.

Gatz M, Johansson B, Pedersen N, Berg S, Reynolds C. A cross-national self-report measure of depressive symptomatology. International Psychogeriatrics. 1993; 5:147-156. [PubMed: 8292768]

Gatz M, Pedersen NL, Plomin R, Nesselroade JR, McClearn GE. Importance of shared genes and shared environments for symptoms of depression in older adults. Journal of Abnormal Psychology. 1992; 101:701-708. [PubMed: 1430610]

Gold CH, Malmberg B, McClearn GE, Pedersen NL, Berg S. Gender and health: A study of older unlike-sex twins. Journals of Gerontology: Series B: Psychological Sciences and Social Sciences. 2002; 57B:S168-S176.

Hawkley LC, Cacioppo JT. Loneliness and pathways to disease. Brain, Behavior, and Immunity. 2003; 17:S98-S105.

Jansson M, Gatz M, Berg S, Johansson B, Malmberg B, McClearn GE, et al. Pedersen NL. Gender differences in heritability of depressive symptoms in the elderly. Psychological Medicine. 2004; 34:471-479. [PubMed: 15259832]

Johnson W, Krueger RF. Genetic effects on physical health: Lower at higher income levels. Behavior Genetics. 2005a; 35:579-590. [PubMed: 16184486]

Johnson W, Krueger RF. Higher perceived life control decreases genetic variance in physical health: Evidence from a national twin study. Journal of Personality and Social Psychology. 2005b; 88:165-173. [PubMed: 15631582]

Kremen WS, Thompson-Brenner H, Leung YJ, Grant MD, Franz CE, Eisen SA, et al. Lyons MJ. Genes, environment, and time: The Vietnam Era Twin Study of Aging (VETSA). Twin Research and Human Genetics. 2006; 9:1009-1022. [PubMed: 17254445]

Lykken DT, Bouchard TJ, McGue M, Tellegen A. The Minnesota Twin Family Registry: Some initial findings. Acta Gemellogicae et Medicae. 1990; 39:35-70.

McClearn GE, Johansson B, Berg S, Pedersen NL, Ahern F, Petrill SA, Plomin R. Substantial genetic influence on cognitive abilities in twins 80 or more years old. Science. 1997; 276:1560-1563. [PubMed: 9171059]

McGue M, Christensen K. Genetic and environmental contributions to depression symptomatology: Evidence from Danish twins 75 years of age and older. Journal of Abnormal Psychology. 1997; 106:439-448. [PubMed: 9241945]

McGue M, Christensen K. Social activity and healthy aging: A study of aging Danish twins. Twin Research and Human Genetics. 2007; 10:255-265. [PubMed: 17564515]

McGue M, Hirsch B, Lykken DT. Age and the self-perception of ability: A twin study analysis. Psychology and Aging. 1993; 8:72-80. [PubMed: 8461118]

Neiderhiser JM, Lichtenstein P. The Twin and Offspring Study in Sweden: Advancing our understanding of genotype-environment interplay by studying twins and their families. Acta Psychologica Sinica. 2008; 40:1116-1123.

Osler M, McGue M, Lund R, Christensen K. Marital status and twins' health and behavior: An analysis of middle-aged Danish twins. Psychosomatic Medicine. 2008; 70:482-487. [PubMed: 18480194]

Owens IPF. Ecology and evolution: Sex differences in mortality rate. Science. 2002; 297:2008-2009. [PubMed: 12242430]

Radloff LS. The CES-D Scale: A self-report depression scale for research in the general population. Applied Psychological Measurement. 1977; 1:385-401.

Rosenthal, R. Meta-analytic procedures for social research (Rev ed). Newbury Park, CA: Sage; 1991.

Rosenthal R, DiMatteo MR. Meta-analysis: Recent developments in quantitative methods for literature reviews. Annual Review of Psychology. 2001; 52:59-82.

Shanahan MJ, Hofer SM. Social context in gene-environment interactions: Retrospect and prospect. Journals of Gerontology: Series B: Psychological Sciences and Social Sciences. 2005; 60B(Special Issue 1):65-76. 
Schmalhausen, I. Factors of evolution: The theory of stabilizing selection. Philadelphia: The Blakiston Company; 1946.

Schoeneborn, CA.; Heyman, KM. Department of Health and Human Services (DHHS). National Health Statistics Reports. Hyattsville, MD: 2009. Health characteristics of adults aged 55 years and older: 2004-2007.

Velozo CA, Byers KL, Wang Y, Joseph BR. Translating measures across the continuum of care: Using Rasch analysis to create a crosswalk between the Functional Independence Measure and the Minimum Data Set. Journal of Rehabilitation Research and Development. 2007; 44:467-478. [PubMed: 18247243] 


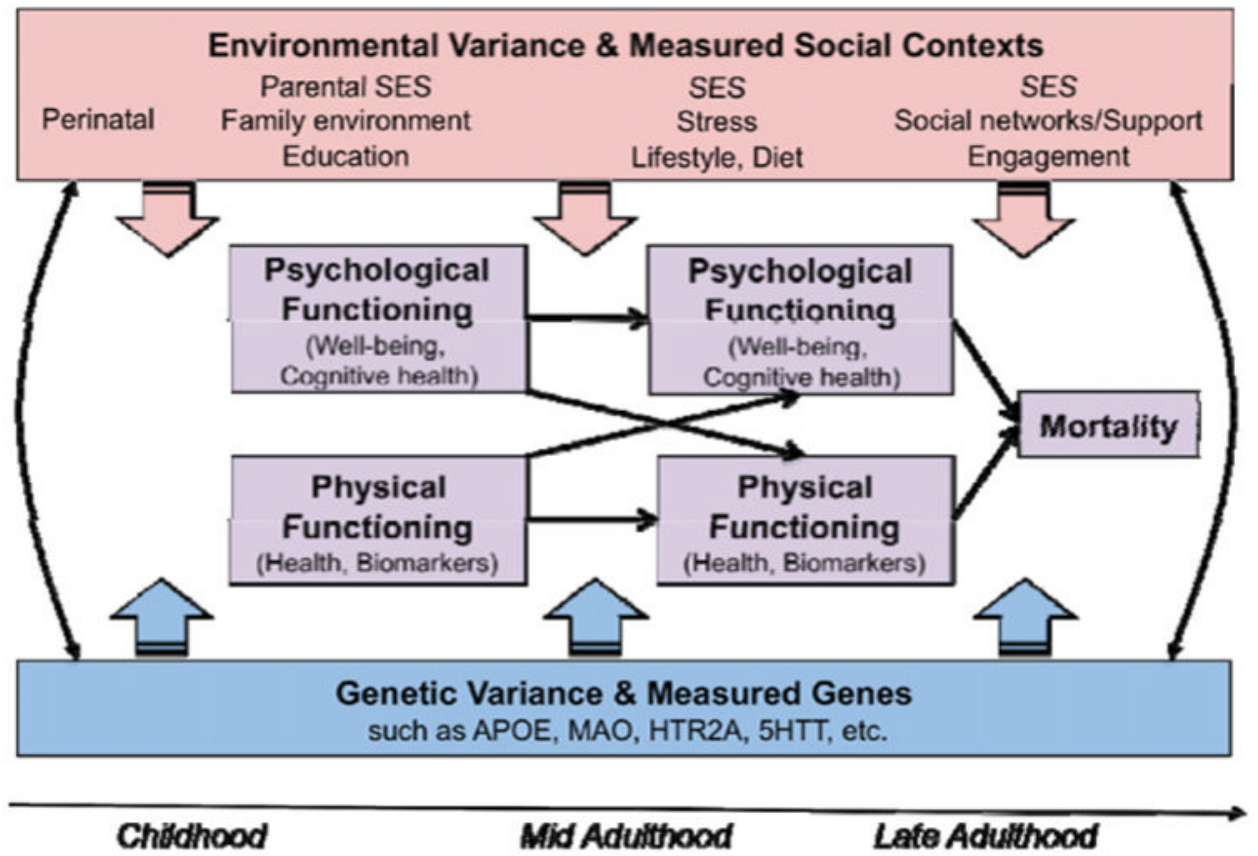

Figure 1.

(Colour online) Heuristic model forming basis of investigation of gene-environment interplay in late-life functioning. 


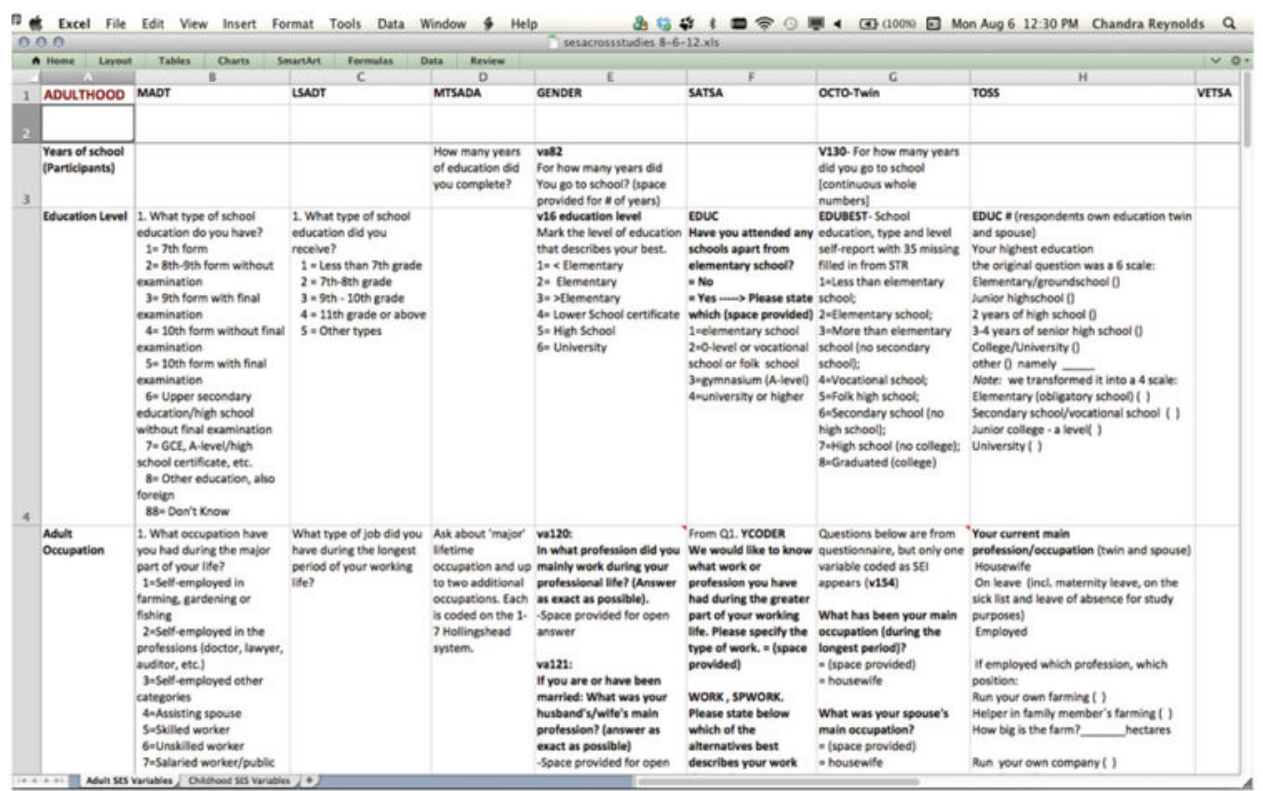

Figure 2.

(Colour online) Example spreadsheet to identify common items: A snapshot of adult SES measures. 


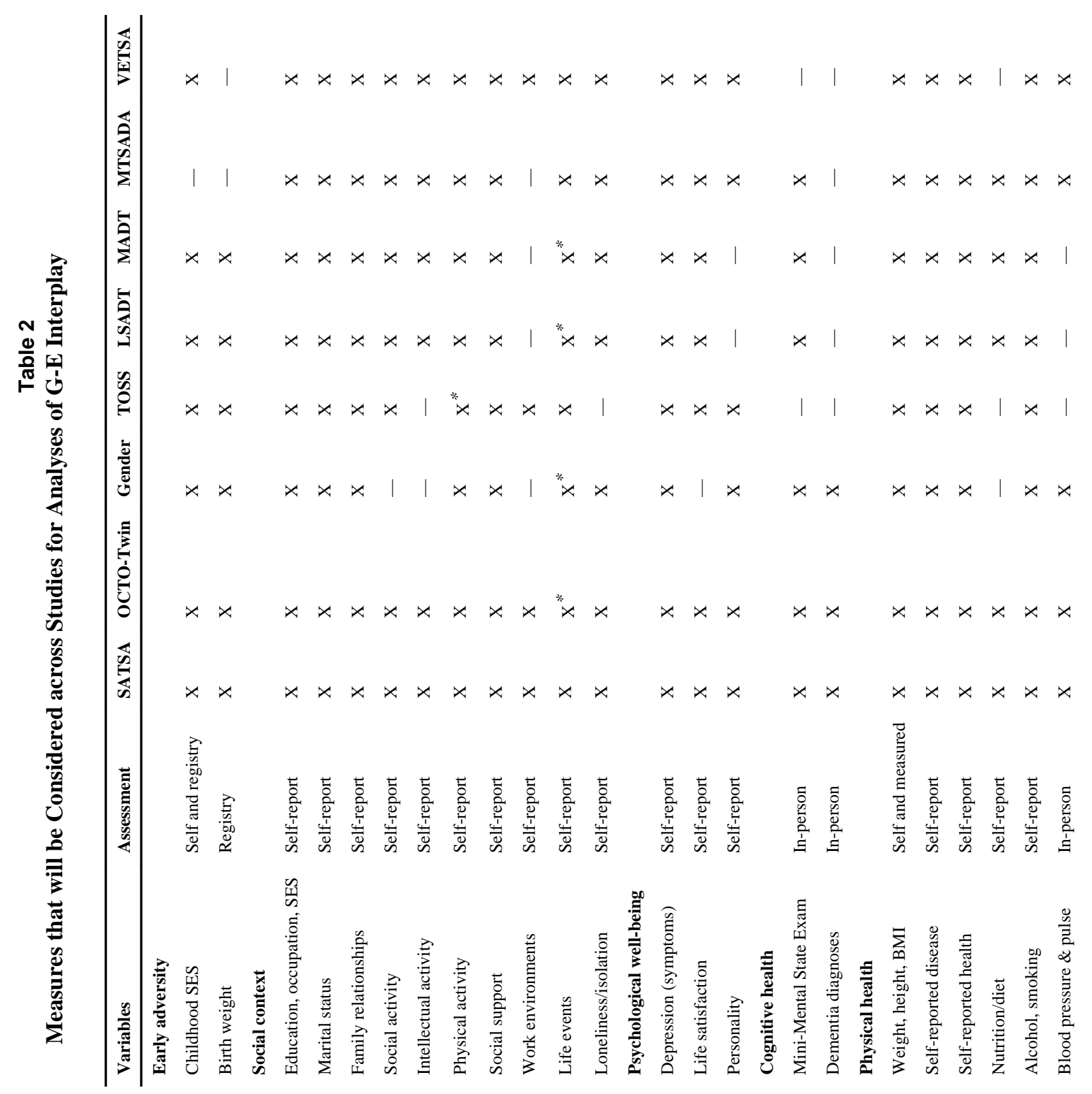




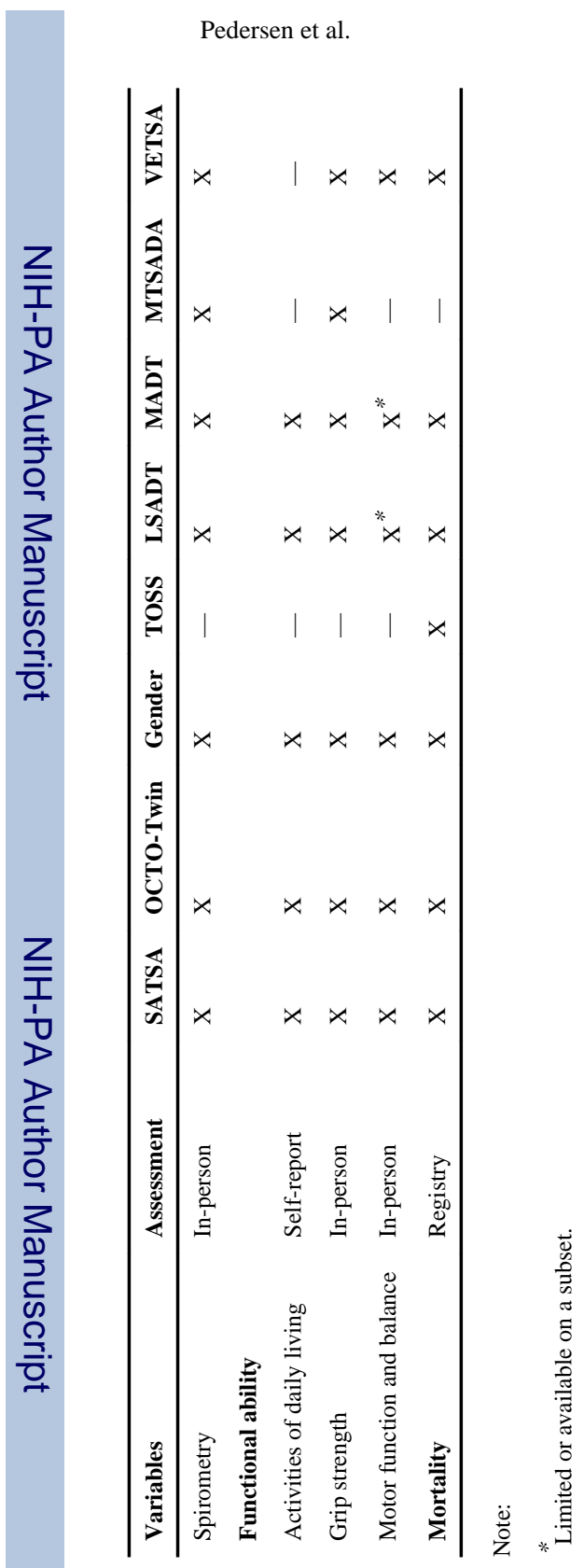

Page 15

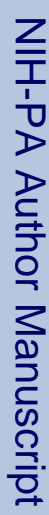

Twin Res Hum Genet. Author manuscript; available in PMC 2013 July 03. 